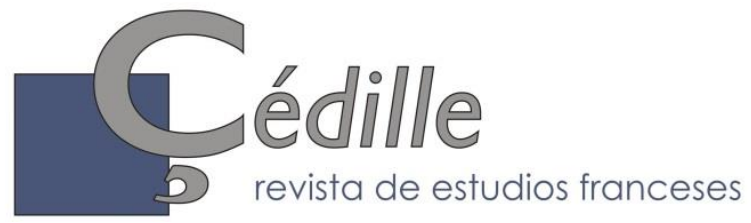

\title{
La réception des femmes poètes de la Belle Époque en France et en Espagne. Présentation
}

\author{
Flavie FouCHARD \\ Universidad de Sevilla \\ flaviefouchard@us.es \\ https://orcid.org/0000-0002-9895-8203
}

\author{
Andrea SCHELLINo \\ Università Roma Tre \\ andrea.schellino@yahoo.it \\ https://orcid.org/0000-0002-7038-130X
}

Alors que plusieurs études et anthologies ont été consacrées aux femmes poètes françaises du XIX ${ }^{\mathrm{e}}$ siècle (comme Femmes poètes du XIX siècle. Une anthologie, sous la direction de Christine Planté, 2010), et plus particulièrement aux poétesses de la Belle Époque (dont la thèse de Patricia Izquierdo sur Les Conditions et modalités de l'essor de la poésie féminine d'expression française à la Belle Époque, soutenue à l'Université Bordeaux III en 2004), la fortune de cette vaste production poétique reste à étudier. Le travail d'Adrianna M. Paliyenko (Genius Envy. Women Shaping French Poetic History, 1801-1900, 2016 ${ }^{1}$ ), axé sur l'émergence de la notion de "génie féminin » et sur son accueil tout au long du $\mathrm{XIX}^{\mathrm{e}}$ siècle, ne concerne qu'en moindre mesure la période 1871-1914. Le volume dirigé en 2019 par Wendy Prin-Conti sur l'héritage des femmes poètes de la Belle Époque (Les Femmes poètes de la Belle Époque: heurs et malheurs d'un héritage) a montré la vitalité de cette tradition littéraire encore mal connue.

En prolongeant et en renouvelant ces approches, notre dossier braque les projecteurs sur la dimension européenne des œuvres des femmes poètes françaises de la Belle Époque, à travers les cas emblématiques de leur réception en France et en Espagne. Plusieurs contributions prennent ainsi en considération la fonction de la presse dans la diffusion de ce corpus poétique. Véhicules de résistances et de polémiques, les journaux et les revues littéraires témoignent néanmoins de la lente reconnaissance des femmes poètes.

Les documents iconographiques, et notamment la photographie, tiennent un rôle de premier plan dans ce moment clef de l'affirmation des femmes dans la république des lettres. Comme l'observe Annie Metz, le portrait photographique permet dès 1850 d'accompagner aussi bien le développement des mouvements féministes que la représentation des femmes de lettres. Au tournant du siècle, il

\footnotetext{
${ }^{1}$ Traduit par Nicole G. Albert et paru en 2020 aux Presses universitaires de Rouen et du Havre sous le titre d'Envie de génie. La contribution des femmes à la poésie française (XIXe siècle).
} 
atteste «l'évolution du statut social des femmes, en les montrant au travail, exerçant des métiers jusqu'alors exclusivement masculins ou pratiquant tous les sports » (Metz, 2017 : 1128). Certaines contributions de notre dossier font ainsi la part belle à ce mode de représentation qui est alors en passe de devenir crucial dans les nouvelles stratégies de construction d'une figure auctoriale.

En Espagne, l'article « Escritoras francesas contemporáneas », paru dans Esos Mundos en septembre 1911, et accompagné de portraits de poétesses, montre que l'essor de la poésie féminine française n'est pas passé inaperçu à l'étranger. Cet accueil, largement méconnu, constitue le second axe de notre recueil. De quelle considération ont joui ces écrivaines hors de France ? Ont-elles été considérées de la même manière que dans leur patrie, c'est-à-dire sans réelle individualité et comme représentantes du «romantisme féminin » (Maurras, 1905) ? Ou ontelles réussi à sortir des caractérisations de la littérature féminine ? Leurs œuvres ont-elles été traduites, à l'époque, ou ont-elles simplement nourri la curiosité autour du monde littéraire français ? Le présent recueil essaie d'apporter quelques éléments de réponse à toutes ces questions.

En ouverture du dossier, Nelly Sanchez analyse le cas singulier du Massacre des Amazones (1899) de Han Ryner (pseudonyme d'Henri Ner), pamphlet antiféministe sous-titré : "Études critiques sur deux cents bas-bleus contemporains », et d'abord publié dans la presse, qui dresse une typologie des femmes de lettres. L'auteure montre que, mutatis mutandis, cet ouvrage misogyne, écrit au vitriol, a favorisé la reconnaissance publique des femmes poètes en leur donnant une visibilité parfois inespérée au sein d'un panorama critique centré sur la production masculine.

Wendy Prin-Conti confronte les portraits photographiques des poétesses et les représentations des poètes masculins dans la presse française entre 1908 et 1914, et en particulier dans Les Annales politiques et littéraires, Femina et Comoedia. La chronologie proposée par la contributrice signale un point d'inflexion dans la critique : selon elle, «les comptes rendus consacrés aux femmes poètes cessent d'être systématiquement à charge ». L'émancipation de la « tutelle masculine » dans le domaine des représentations photographiques est tout aussi progressive que dans la critique.

Nicole Laval-Turpin étudie la première fortune critique d'Hélène Picard, lauréate du prix Femina en 1904. La poétesse contribue à faire changer le regard des critiques sur la poésie féminine en publiant L'Instant éternel en 1907. La contributrice s'attache particulièrement aux stratégies employées par la revue Femina pour présenter Hélène Picard, qui allie savamment citations de poèmes, portraits photographiques et articles de fond.

Carmen Ramírez Gómez analyse la présence de Marie Dauguet, collaboratrice du Mercure de France qui occupe une place de choix dans les Muses d'aujourd'hui de Jean de Gourmont, dans la presse française du tournant du siècle. L'étude propose une mise en perspective de la portée de l'œuvre de la poétesse dans la littérature française de l'époque, et dans les lettres féminines en par- 
ticulier, marquées par les tensions et les débats quant à la place des femmes en littérature.

Après ces études sur la présence des femmes dans la presse française, notre dossier se consacre au domaine espagnol. Tout d'abord, Isabel Clúa Ginés prend en considération l'accueil critique de l'œuvre de Lucie Delarue-Mardrus tout en insistant sur le fait qu'il est indissociable de sa personnalité de femme de lettres. À partir de ce constat, elle souligne son exemplarité dans la construction de la figure d'écrivaine au début du $\mathrm{XX}^{\mathrm{e}}$ siècle : comme les autres poétesses elle se trouve en proie aux difficultés éprouvées par les femmes pour exister en tant qu'auteures littéraires dans la sphère publique.

Les deux contributions qui closent le recueil sont consacrées à Anna de Noailles. Francisco Lafarga examine d'abord l'accueil de la poétesse en son temps et sa réception critique. Il prend ensuite en considération les articles parus en Espagne à l'occasion de sa mort, et en particulier ceux qui fixent l'image d'une poétesse délicate et raffinée. Enfin, l'étude d'Irene Atalaya témoigne de la vitalité de l'œuvre des poétesses de la Belle Époque dans le domaine de la traduction contemporaine : Renée Vivien est la plus traduite mais Anna de Noailles n'est pas en reste. La contributrice analyse ainsi la postérité de l'œuvre d'Anna de Noailles par le biais des traductions en espagnol de Mireia Alonso Ribeiro (Las pasiones y las tumbas, 2011, rééd. 2020) et de Julio Pollino Tamayo (El honor de sufrir, 2018).

\section{RÉFÉRENCES BIBLIOGRAPHIQUES}

Albert, Nicole. G. (2009) : Renée Vivien à rebours. Paris, L'Harmattan.

Bedeschi, Andrea, Adriano Marchetti \& Valentina GosetTI, (2017) : Donne. Poeti di Francia e oltre. Dal Romanticismo a oggi. Borgomanero, Giuliano Ladolfi.

Boutin, Aimée (2004) : «Inventing the "Poétesse": New Approaches to French Women Romantic Poets ». Romanticism on the Net, 29. URL : https://www.erudit.org/fr/revues/ron/2003-n29-30-ron695/007725ar.

EDWARDS, Paul (2009) : «Renée Vivien et la photographie », in Nicole G. Albert (dir.), Renée Vivien à rebours Études pour un centenaire. Paris, L'Harmattan.

EDWARDS, Paul (2016) : «1900-1908 : la vision de l'écrivain », in Soleil noir, Rennes, PUR.

HEINICH, Nathalie (2012): De la visibilité. Excellence et singularité en régime médiatique. Paris, Gallimard.

IZquiERDO, Patricia (2009) : Devenir poétesse à la Belle Époque (1900-1914). Étude littéraire, historique et sociologique. Paris, L'Harmattan.

KULESSA, Rotraud von (2011): Entre la reconnaissance et l'exclusion. La position de l'autrice dans le champ littéraire en France et en Italie à l'époque 1900. Paris, Honoré Champion.

LASSERRE, Audrey (2009): «Les femmes du XX $X^{\mathrm{e}}$ siècle ont-elles une histoire littéraire ? ». Cahier du CERACC, 4, 38-54. 
LEVy, Gayle A. (2002) : «La Chambre du poète », in Christine Planté (éd.), MasculinFéminin dans la poésie et les poétiques du XIX siècle. Lyon, Presses Universitaires de Lyon, 449-457.

MaCDONALD, Myra (1995) : Representing Women: Myths of Femininity in the Popular Media. London, etc., Arnold.

MAURRAS, Charles (1905) : L'Avenir de l'intelligence, suivi d'Auguste Comte. Le romantisme féminin-Mademoiselle Monk. Paris, Albert Fontemoing.

MESCH, Rachel (2013) : Having It All in the Belle Époque : How French Women's Magazines Invented the Modern Woman. Stanford University Press. ProQuest Ebook Central,

URL : https://ebookcentral.proquest.com/lib/uses/detail.action?docID=1322671.

Metz, Annie (2017) : «Photographie », in Christine Bard (dir.) Dictionnaire des féminismes. Paris, PUF, 1127-1129.

MetZ, Annie \& Florence Rochefort (2010) : Photo / Femmes / Féminisme. 1860-2010. Paris, Collection de la bibliothèque Marguerite Durand, Paris bibliothèque éditions.

MeIzOz, Jérôme (2007) : Postures littéraires. Mises en scène modernes de l'auteur. Genève, Slatkine.

OTTO, Elizabeth \& Vanessa Rocco [(dir.] (2011) : The New Woman International: Representations in Photography and Film from the 1870s Through the 1960s. Ann Arbor, University of Michigan Press and the University of Michigan Library.

PaliyenKo, Adrianna M. (2016) : Genius Envy. Women Shaping French Poetic History, 1801-1900. Penn State University Press.

PARINET, Elisabeth (1991) : «L'édition littéraire, 1890-1914 », in Roger Chartrier \& Henri-Jean Martin (dir.), Histoire de l'édition française Le livre concurrencé 1900-1950. Paris, Fayard, 161-209.

PlantÉ, Christine (1998): Femmes poètes du XIX siècle: Une anthologie. Lyon, Presses universitaires de Lyon.

Planté, Christine (2002) : «Quand Je est un(e) autre », in Christine Planté (dir.), Masculin-Féminin dans la poésie et les poétiques du XIXe siècle. Lyon, Presses universitaires de Lyon, 81-100.

Planté, Christine (2003) : «La place des femmes dans l'histoire littéraire : annexe, ou point de départ d'une relecture critique? ». Revue d'histoire littéraire de la France, 103 : 3, 655-668.

Planté, Christine (2010) : «La Petite Søur de Balzac. Vingt-cinq ans après ». FabulaLhT, 7, URL : http://www.fabula.org//ht/7/plante.html, 15 avril 2018.

PlANTÉ, Christine (2011) : "La place problématique des femmes poètes », in Martine Reid (dir.), Les Femmes dans la critique et l'histoire littéraire. Paris, Honoré Champion, 55-72.

Planté, Christine (2012). : Compte rendu de Patricia Izquierdo, Devenir poétesse à la Belle Époque (1900-1914). Clio. Femmes, genre, histoire, 36, URL: http://journals.openedition.org/clio/10906. 
PINSON, Guillaume (2009) : «La femme masculinisée dans la presse mondaine française de la Belle Époque». Clio. Histoire, femmes et sociétés, 30 URL: http://journals.openedition.org/clio/9471.

Prin-ContI, Wendy (dir.) (2019): Les Femmes poètes de la Belle Époque : heurs et malheurs d'un héritage. Paris, Honoré Champion.

REID, Martine et al. [dir.] (2020) : Femmes et littérature : une histoire culturelle. Paris, Gallimard.

SAINT-MARTin, Monique de (1990) : «Les femmes écrivains et le champ littéraire ». Actes de la recherche en sciences sociales, 83, 52-56.

TOURET, Michèle (2010) : «Où sont-elles ? Que font-elles ? La place des femmes dans l'histoire littéraire. Un point de vue de vingtièmiste». Fabula-LhT, 7, URL: http://www.fabula.org/lht/7/touret.html. 